\title{
Swarm SCARF Dedicated Lithospheric Field Inversion chain
}

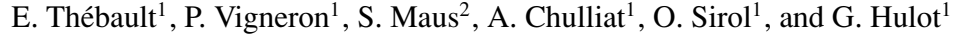 \\ ${ }^{1}$ Equipe de Géomagnétisme, Institut de Physique du Globe de Paris, Sorbonne Paris Cité, Université Paris Diderot, \\ UMR CNRS 7154, 1 rue Jussieu F-75005 Paris, France \\ ${ }^{2}$ Geophysical Data Center, NOAA, Boulder, Colorado, U.S.A.
}

(Received March 31, 2013; Revised July 10, 2013; Accepted July 22, 2013; Online published November 22, 2013)

\begin{abstract}
The forthcoming Swarm satellite mission is a constellation of three satellites dedicated to the study of the geomagnetic field. The orbital characteristics of the mission, which includes a pair of satellites flying side by side, has prompted new efforts in data processing and modeling. A consortium of several research institutions has been selected by the European Space Agency (ESA) to provide a number of Level-2 data products which will be made available to the scientific community. Within this framework, specific tools have been tailor-made to better recover the lithospheric magnetic field contribution. These tools take advantage of gradient properties measured by the lower pair of Swarm satellites and rely on a regional modeling scheme designed to better detect signatures of small spatial scales. We report on a processing chain specifically designed for the Swarm mission. Using an End-to-End simulation, we show that the tools developed are operational. The chain generates a model that meets the primary scientific objectives of the Swarm mission. We also discuss refinements that could also be implemented during the Swarm operational phase to further improve lithospheric field models and reach unprecedented spatial resolution.
\end{abstract}

Key words: Lithospheric magnetic field, space magnetometry, Swarm.

\section{Introduction}

Large magnetic anomaly field features with spatial dimensions of more than a few hundred kilometers can be inferred from Low Earth Orbiting (LEO) satellites. These length scales are typically dominated by the magnetic field created by the spatial variation in structure and composition of the Earth's crust and upper mantle (Thébault et al., 2010 for a review). The previous CHAMP (Reigber et $a l ., 2002)$ satellite mission allowed impressive progress towards mapping the Earth's lithospheric magnetic field (e.g., Maus et al., 2008), understanding their geological sources (e.g., Hemant and Maus, 2005), and inferring other physical quantities such as the equivalent global Curie depth (Purucker et al., 2002) or the heat flow anomalies of the Earth's crust in remote regions (e.g., Fox-Maule et al., 2005). The Earth's lithospheric field at large wavelengths $(>400 \mathrm{~km})$ is conceptually simple because mostly static in time (e.g., Thébault et al., 2009). Unfortunately, it is also one of the weakest detectable sources at satellite altitudes and is masked by all other constituents of the total Earth's magnetic field (e.g., Hulot et al., 2007). For instance, it represents about $0.01 \%$ of the total Earth's magnetic field strength at $400 \mathrm{~km}$ height (with a root mean square of about $3.8 \mathrm{nT}$ using the NGDC-720 model; Maus, 2010) and its magnitude rarely exceeds $20 \mathrm{nT}$.

Planetary magnetic fields are often expressed in terms of a finite series of spherical harmonics ( $\mathrm{SH})$ to a maximum

Copyright (c) The Society of Geomagnetism and Earth, Planetary and Space Sciences (SGEPSS); The Seismological Society of Japan; The Volcanological Society of Japan; The Geodetic Society of Japan; The Japanese Society for Planetary Sciences; TERRAPUB.

doi:10.5047/eps.2013.07.008 expansion degree. After ten years of the CHAMP mission, the most recent models describing the magnetic field of the lithosphere, such as the MF7 model (an upgrade of Maus et al., 2008; http://geomag.org/models/MF7.html) expanded to SH degree 133, and the CHAOS-4 model (an upgrade of Olsen et al., 2010a) expanded to SH degree 100, agree reasonably well up to degree 85 , thus providing a robust bound of about $470 \mathrm{~km}$ on the maximum horizontal spatial resolution. The geometrical attenuation with altitude and the prominence of external fields at satellite level are the two main factors limiting our ability to reach higher SH degrees and thus to detect smaller scale structures. However, for equivalent altitude datasets, the differences between MF7 and CHAOS-4 mainly lie in the way the different models deal with source field separation and, more specifically, with the rapidly time-varying external fields.

Two approaches can indeed be followed for the analysis of magnetic field measurements from space. The first one, referred to as the comprehensive inversion (CI) approach, attempts to solve for all known magnetic field contributions through a grand inversion (e.g., Sabaka and Olsen, 2006; Sabaka et al., 2013; Olsen et al., 2010a). This approach requires some assumptions about the geometry of the dominant source fields to guide the model parameterization. Because of the time needed to properly sample the Earth's surface and because of the satellite orbital precession through local time (LT), the CI approach relies on mathematical models describing the sources only on average in space and time. For the recent CHAMP mission, the complete coverage in space and LT was achieved after about 130 days (Olsen et al., 2010b, figure 8). As a result, in such models the external magnetic fields with higher time frequen- 
cies may overlap with the small scale and weak lithospheric fields so that both contributions can be difficult to identify without ambiguity (e.g., Olsen et al., 2010c).

One may follow a second approach, referred to as the sequential approach, and focus on one specific constituent such as the lithospheric magnetic field (e.g., Maus et al., 2008). The ambiguity with respect to internal and external field separation can then be partly removed by adding prior information or by selecting measurements in a way that is more optimum. This is a complex and subjective procedure where the investigator's decisions are needed all along the way to isolate the corresponding contributions. This approach successfully led to a suite of lithospheric field models with unprecedented spatial resolution (SH degree 133 for the MF7 model). Even though specific problems related to spectral leakage can arise (Sabaka and Olsen, 2006; Thébault et al., 2012), implying that all SH coefficients are not necessarily well resolved, these models clearly revealed striking regional correlations between magnetic anomalies and continental geological bodies at small spatial scales.

One of the primary goals of the European Swarm satellite mission (Friis-Christensen et al., 2006) is to unveil the lithospheric field signal to the highest possible horizontal spatial resolution. We therefore decided to develop a processing chain dedicated to the production of a lithospheric field model. This so-called 'Dedicated Lithospheric Field Inversion Chain' was proposed within the Swarm SCARF (Satellite Constellation Application and Research Facility, see Olsen et al., 2013) set up by a consortium of several research institutions and selected for implementation by ESA. It will be operated during the mission as one of more than a dozen Level 2 processing chains, which will also include a Comprehensive Inversion chain (producing an independent lithospheric field model, see Sabaka et al., 2013) and two other chains: the Swarm SCARF Dedicated Ionospheric Field Inversion chain (Chulliat et al., 2013) and the Swarm SCARF equatorial electric field inversion chain (Alken et al., 2013) developed by IPGP or in collaboration with NOAA.

Whichever the chosen modeling approach, the general idea is to carefully represent the selected and corrected data by a single set of parameters that allows computing the lithospheric magnetic field values outside the dominion of data. The common approach relies on the SH series expansion that is the most natural solution of the Laplace's equation in the geocentric reference frame. Here we favor a regional modeling scheme which has several advantages. First, it makes it possible to bypass the problem of truncated spherical harmonics that can arguably be suboptimal for the purpose of highlighting small magnetic field wavelengths. Indeed, splitting the global problem into subregions allows high regional spatial resolution to be reached with a manageable number of parameters. Second, correction errors result mostly from transient external magnetic fields that contaminate the data in a different way depending on the location. Regional modeling can adjust to this by treating the noise regionally; it also offers the possibility of constraining the inverse problem depending on how well the regional model parameters are resolved (e.g., Lesur and Maus, 2006). Finally, computing regionally the covariance matrices of the errors for a better understanding of their correlation in space and in time is also possible, something that is demanding in terms of computer resources when building high degree $\mathrm{SH}$ models.

We report below on the Dedicated Lithospheric Field Chain as it was implemented during the preparation phase of the Swarm satellite mission. The block processes were duly tested in the course of the development and we do not discuss the details of the closed-loop simulations that were successfully completed. Rather, we focus on the end-toend simulation and describe in some details the algorithm, its advantages but also its shortcomings. Because the dedicated techniques rely on the operator's judgment rather than unequivocal scientific observations (which often raises some contentious issues), we describe the difficulties we met and the compromises we decided to make. We finally show that the chain is able to produce a lithospheric field model that reaches the Swarm performance criteria (Swarm Level 2 Processing System Consortium, 2013).

\section{Theoretical Background}

Different regional modelling techniques are currently used or under development in the field of geomagnetism (see Schott and Thébault, 2011, for a review). These are based, for instance, on frames of Poisson wavelets (e.g., Maier and Mayer, 2003), slepian functions (e.g., Beggan $e t$ al., 2013), combination of band-limited spherical harmonics (e.g., Lesur, 2006), spherical tesselation (e.g., Stockman et al., 2009), or equivalent dipole source distributions (e.g., Langlais et al., 2004). We choose to rely on the Revised Spherical Cap Harmonic Analysis (R-SCHA, Thébault et al., 2006) technique as this approach was successfully used with the CHAMP magnetic data to build a global model of the Earth's lithospheric field (Thébault, 2006). We briefly restate the basic properties of the modelling strategy we follow. A complete description of the Revised Spherical Cap Harmonic Analysis technique can otherwise be found in Thébault et al. (2004, 2006).

The solution is obtained within a conical domain bounded by two spherical caps of aperture $\theta_{0}$ at altitudes $\epsilon_{1}$ and $\epsilon_{2}$; the surface being closed by the lateral boundary of the cone (see figure 1 in Thébault et al., 2006). The solution for the potential $V_{\mathrm{SCHA}}$ can be expressed in a spherical coordinate system as a sum of two infinite series

$$
\begin{aligned}
V_{\mathrm{SCHA}}(r, \theta, \varphi)= & a \sum_{m=0}^{\infty}\left(\sum_{k=1}^{\infty} \bar{G}_{k}^{m} \bar{\psi}_{k}^{m}(r, \theta, \varphi)\right. \\
& \left.+\sum_{p=1}^{\infty} \bar{G}_{p}^{m} \bar{\Pi}_{p}^{m}(r, \theta, \varphi)\right),
\end{aligned}
$$

where $\bar{\psi}_{k}^{m}=R_{k}(r) e^{i m \varphi} P_{n_{k}}^{m}(\cos \theta)$ are the complex spherical cap harmonics of order $m$ involving the Legendre function $P_{n_{k}}^{m}(\cos \theta)$ of real $n_{k}$ (see Haines, 1985) and $R_{k}(r)$ their radial functions, while $\bar{\Pi}_{p}^{m}=R_{p}(r) e^{i m \varphi} K_{p}^{m}(\cos \theta)$ are the complex harmonics expressed in terms of the conical, or Mehler, functions and $R_{p}(r)$ the real-valued radial functions (see Thébault et al., 2006, equations (18)-(19)), $a$ is the Earth's mean radius and $\theta, \varphi$, and $r$ are respectively the colatitude, the longitude and the radius (in a reference frame 
whose axis is aligned with the axis of the cone), and $\bar{G}^{m}$ the regional parameters for each order $m$ written in their complex form. There are many ways of numerically defining the basis functions, one of which being to impose homogeneous boundary conditions on each of the three surfaces. In the present work, we apply the formalism described in Thébault et al. (2004), where the boundary value problem is solved with Neumann homogeneous boundary conditions on all three surfaces. In practice, the complete solution requires a second potential that is added to the one defined in Eq. (1)

$$
V_{\mathrm{SH}}(r, \theta, \varphi)=a \sum_{n=1}^{\infty}\left(\frac{a}{r}\right)^{n+1} G_{n}^{0} \psi_{n}^{0},
$$

with $\psi_{n}^{0}=P_{n}^{0}(\cos \theta)$ the real-valued zonal spherical harmonic of integer degree $n$, the axis of which coincides with the cone's axis, and $G_{n}^{0}$ the zonal SH Gauss coefficients in the cone's reference frame. This second potential is required to bypass a problem of incompleteness of the potential expressed in Eq. (1) when the geomagnetic field is to be represented (see Thébault et al., 2004). The solution for the magnetic field $\mathbf{B}$ is then obtained considering the gradient of the two potentials

$$
\mathbf{B}=-\nabla\left(V_{\mathrm{SCHA}}+V_{\mathrm{SH}}\right) .
$$

Empirically, we observe that a solution defined by the Neumann boundary conditions converges fast for the vector magnetic field components. This is a desired property when dealing with anomaly fields still possibly containing large spatial scales magnetic field contaminations, which is usually the case when processing real satellite measurements or realistic synthetic Swarm datasets built from SH models. The spherical and spherical cap harmonics both allow an exact representation of any poloidal magnetic field so that the two solutions converge in norm to each other. However, some representation errors invariably occur when the infinite series (Eqs. (1) and (2)) are truncated to finite expansion indices. A regional solution converging fast can represent the magnetic field $\mathbf{B}$ with fewer parameters than a slow converging series expansion. It guarantees a better comparison with the benchmark model during the synthetic validation of the algorithm as it mitigates the problem of truncation errors. There are some disadvantages, though, of combining the Eq. (1) with (2). In particular, the basis functions are not mutually orthogonal within the conical domain (Thébault et al., 2004). This leads to a non-diagonal design matrix where cross-talking between parameters implies some reorganization of their estimated values when changing the series truncation. In general, this is not ideal for carrying out spectral analysis of the magnetic field signal or for isolating a single model parameter. For the purpose of this work, this is a minor issue because we do not aim at interpreting the values of each regional parameter independently and because we only deal with high spatial density satellite data. In such a case, the design matrix, although not diagonal, is never dense. The inverse problem is numerically stable.

Each type of functions (Mehler or Legendre) has specific properties (Thébault et al., 2006). In particular, the maximum expansion index $k_{\max }$ of series in Eq. (1) can be asymptotically related to the degree $n_{k}$ of the Legendre functions (Haines, 1985; Thébault et al., 2006)

$$
n_{k} \approx\left(2 k_{\max }-m-1 / 2\right) \frac{180}{2 \theta_{0}}-\frac{1}{2},
$$

which may be expressed in terms of the minimum length scale $\gamma$ of the magnetic signal (Backus et al., 1996, section 3.6.3)

$$
\gamma \simeq \frac{2 \pi(a+\varepsilon)}{n_{k}+1 / 2}
$$

where $\varepsilon$ is the considered altitude. For $k_{\max }=15$ and $\theta_{0}=8^{\circ}$, for example, we find $\gamma \simeq 130 \mathrm{~km}$ at $400 \mathrm{~km}$ altitude; these parameters are the ones selected for the present regional modeling.

\section{The Dedicated Lithospheric Field Inversion (DLFI) Chain}

The full chain is represented in Fig. 1. The Swarm Level $1 \mathrm{~b}$ data are first selected according to specific criteria and are corrected for various sources fields. As already discussed, this step is crucial for deriving the lithospheric model. It involves four processes (Process 1 to Process 4 in Fig. 2) that require auxiliary information and operator's decisions. The selected and corrected measurements are then iteratively processed with the R-SCHA modeling. The data are distributed into geographical bins (Process. 5.1) corresponding to each spherical cap covering the Earth's surface and transformed into the cap's reference frame (Process 5.2). The inverse problem is solved, and the regional parameters stored, after building the design matrix (Process 6.1) and solving the system by Singular Value Decomposition (SVD in Process 6.2). Once the regional inversions are completed for all caps, the regional parameters are used to predict the lithospheric vector field values on the nodes of a Gauss-Legendre grid (Process 7) covering the Earth's surface at a constant altitude. These grid values are finally converted into a unique set of spherical harmonics Gauss coefficients either by a fast spherical transform of the vertical magnetic field component or by a linear inversion of the horizontal and vertical components (Process 8). The dedicated lithospheric field model is the output product of the sequence of these numerical processes. Each process is described in more details in the following.

\subsection{Data selection}

For this simulation, we use only the vector Level $1 \mathrm{~b}$ data simulated for the lowest pair of satellites Swarm A and Swarm B (Olsen et al., 2013) since the third satellite, Swarm C, is expected to be too high to detect the fine lithospheric field structures. Nevertheless, its data could prove useful for the chain in case of a failure of Swarm A or $\mathrm{B}$ and all data selection and correction procedures described below were also made operational for processing Swarm C magnetic field measurements. Considering that vector magnetometers may suffer from malfunctions, the chain was also adapted to ingest scalar magnetic measurements if required.

More than four years of simulated vector Swarm A and B data are selected between a simulated time period extend- 


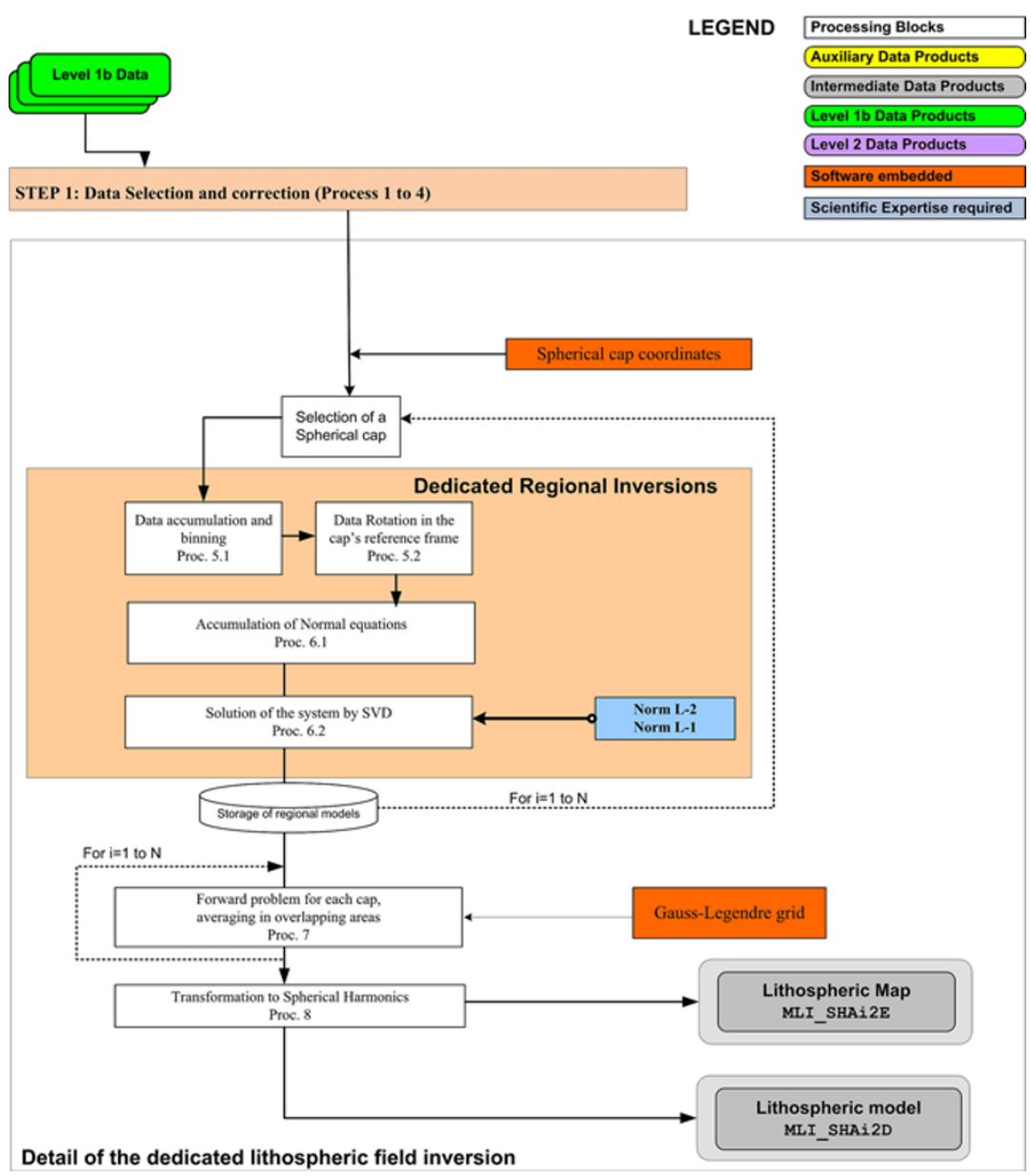

Fig. 1. General flowchart of the six main processes run to generate a lithospheric field model from the Level $1 \mathrm{~b}$ magnetic field measurements of the simulated Swarm satellite mission.

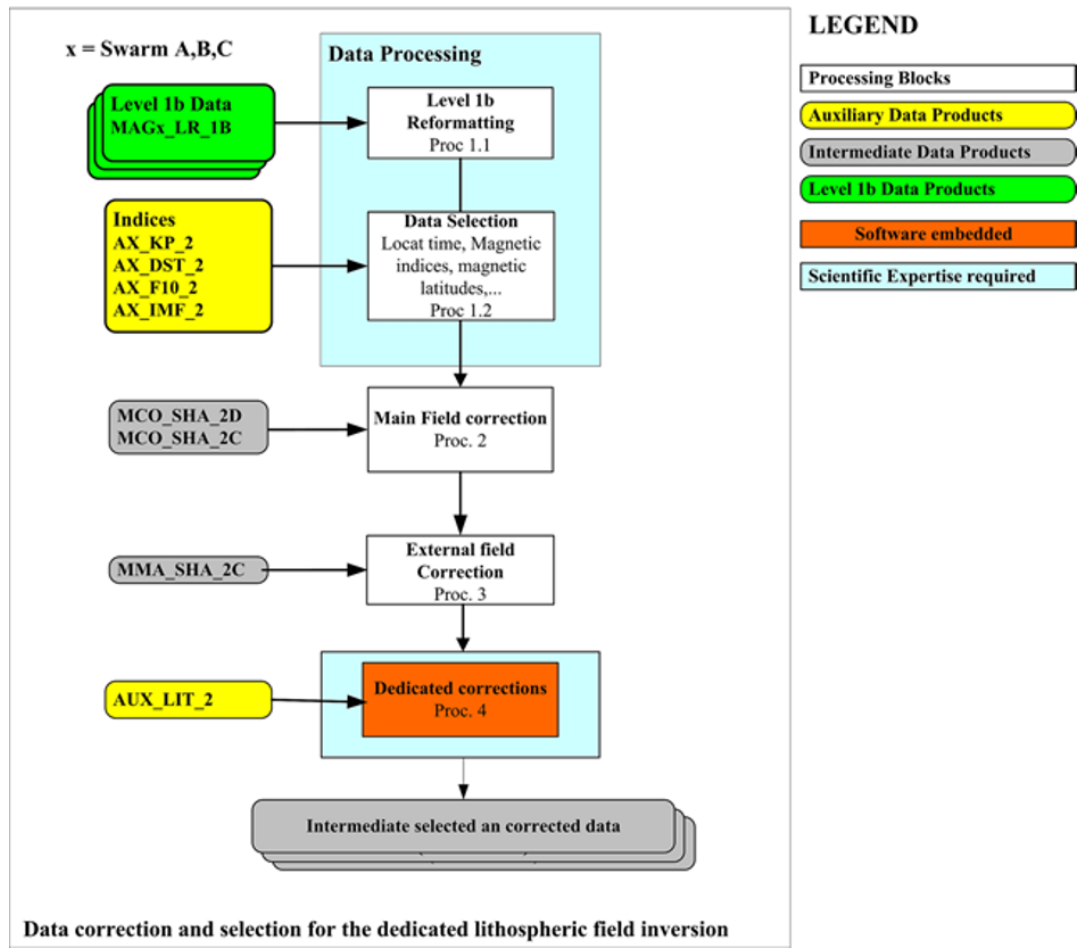

Fig. 2. This flow chart provides the sequence of the processing steps allowing to isolate the magnetic field contributions from the Earth's lithosphere. 
ing from July 1998 to December 2002. These data are subsampled every 10 seconds, corresponding to a spacing of about $70 \mathrm{~km}$ along the satellite track at $400 \mathrm{~km}$ altitude. This ensures that no aliasing occurs because of magnetic field contributions at small wavelengths (recall that the minimum spatial wavelength detected by the regional model is $130 \mathrm{~km}$ with the current setting). Although the synthetic data do not contain toroidal external fields from field aligned currents, we account for the different behavior of the external field with latitudes by separating the vector data in two sets, one for mid-latitudes $\left(-52^{\circ}\right.$ to $52^{\circ}$ magnetic latitudes), and one for polar regions $\left(>52^{\circ}\right.$ and $\left.<-52^{\circ}\right)$. In addition, we allow an overlap of $10^{\circ}$ between mid and polar latitude regions (see also Maus et al., 2008, for instance) so that the provisional selected data for both sets range between $-57^{\circ}$ to $57^{\circ}$ magnetic latitude for the mid-latitude set and above $| \pm 47|$ for the polar set. As discussed below, this overlap is introduced to reduce correction errors caused by dedicated corrections applied along the satellite tracks. The mid-latitude data are further selected at 21:00 to 5:00 local time (LT) in order to minimize the contributions from the ionospheric $S q$ field. All LT are considered for the polar data, provided they are outside the sunlit area $\left(-10^{\circ}\right.$ below horizon). We use the Dst index to lower the effect of the magnetic field generated by the ring current and select the data corresponding to Dst values lower than $\pm 20 \mathrm{nT}$ and to Dst variations smaller than $10 \mathrm{nT}$ over the previous three hours. We also apply a selection criterion based on the $K p$ index to keep data corresponding to a global low level of geomagnetic activity. The data corresponding to a $K p$ index larger than 2, and to $K p$ variations larger than 2 over the previous three hours, are rejected. For this study, the selected Dst threshold is relatively large. We argue that rejecting many satellite data because of a high level of magnetic activity does not necessarily reduce the noise in the final model, as the data error variance is proportional to the ratio $v / M$, where $v$ is the variance of the external field and $M$ the number of available orbits over a given longitude (e.g., Lesur et al., 2013). It is important to define an acceptable trade-off between the number of available satellite tracks and the level of external field activity, more particularly when processing the data on regional scales because the local functions require a dense data coverage in space.

The selection procedure we applied is not exhaustive. The process 1.2 (see Fig. 2) contains more data selection modules than the ones activated for this simulation. Among other selection criteria we included a processing block based on the measure of the Interplanetary Magnetic Field (IMF), whose value is related to the solar wind activity. We included also a selection block based on the ap index, which measures the general magnetic activity at the planetary scale, and a selection block based on quality flags such as the on/off status of the star camera. Other selection blocks are likely to be added as more and more experience is gained on the Swarm satellite data during the calibration/validation and operational phases.

\subsection{Data correction for the main and external fields}

We developed two families of magnetic field corrections for the selected vector data. The first family relies on explicit models for the main field, its secular variation, and the magnetospheric field, (processes 2 and 3 in Fig. 2), all expanded in spherical harmonics. These input models are provided by the other Swarm processing chains (see Olsen et $a l ., 2013$ ). Note that the data are not corrected for the ionospheric field, which is assumed minimum at night-times (see Chulliat et al., 2013). The second family of correction is based on more empirical processes, performed either at the global scale, or along the satellite orbits (process 4). The settings of this suite of corrections depend on the analysis of the data residual after the completion of processes 2 and 3.

Within the overall process proposed by the consortium (see Olsen et al., 2013), the dedicated lithospheric field chain is initiated as soon as the models for other source fields are available for use. The main field correction can be performed using either the output of the comprehensive inversion chain (Sabaka et al., 2013) or that of the dedicated core field chain (Rother et al., 2013). In principle, there is a better consistency between the data selection detailed in the previous section and the data selection applied in the dedicated chain for the core field (Lesur et al., 2010; Rother et al., 2013), particularly at night times. This consistency is important if one keeps in mind that differences in data selection are acknowledged to be one of the major sources of discrepancies between main field models yet parameterized in similar ways (e.g., Finlay et al., 2010). In the course of the Swarm preparation phase, we tested the influence of each of the input main field models and the results were similar. In this paper, the solution is obtained with the vector data corrected with the MCO_SHAi2D (the output of the core field dedicated inversion, see Fig. 2) for the main field model and its secular variation up to SH degree 15 . Since the synthetic Swarm vector measurements were generated using a secular variation model up to $\mathrm{SH}$ degree 20 (Olsen et al., 2013), the secular variation is only partly corrected for. The corrections for the external magnetospheric sources are made using the MMA_SHAi2C model (Sabaka et al., 2013; see also Fig. 2). This model provides coefficients for the primary magnetospheric field to SH degree 3 and a maximum order 1 with a time step of 1.5 hours. The induced counterpart is also defined every 1.5 hours but to maximum degree and order 5. This space and time resolution is lower compared to the benchmark magnetospheric field model used to generate the synthetic data. This represents another source of significant correction error.

Figure 3 displays the residual field for the radial component after correcting explicitly for the main and magnetospheric fields (the known synthetic lithospheric field are removed in this picture for the purpose of illustration). Thanks to the availability of the benchmark model used to compute the synthetic data, we know that these large-scale residuals are dominated by residual magnetospheric contributions. Another important source of errors comes from the ionospheric field at night-times (particularly its induced counterpart), which was not corrected. The secular variation error of the main field is of secondary importance. In total, the correction errors accumulated using SCARF scientific products reach almost $\pm 20 \mathrm{nT}$ in two lobes located near the South geographic pole.

We next identify the Swarm A and B data with the com- 

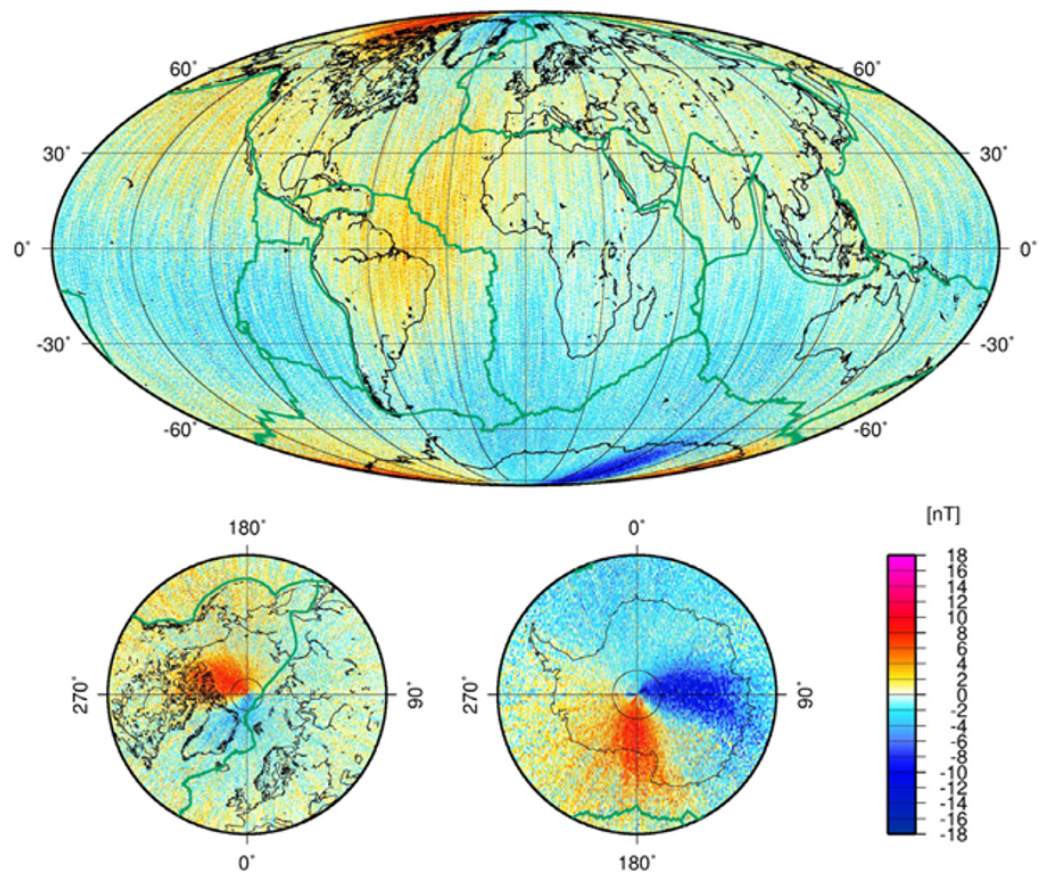

$[\mathrm{nT}]$

Fig. 3. Radial component of the residual field after correction of the Swarm A and B simulated measurements for a main and a magnetospheric field model. The known lithospheric field is removed for this illustration. Projection is Mollweide.

mon reading times and built up a set of so-called gradient data. The terminology of gradient is misleading here as it refers to a point to point difference between the measured data across the two orbits of the lowest pair of satellites (Swarm A and B). This difference is expressed in the same units as the vector measurements (in nT), which avoids the unnecessarily complication of solving the inverse problem with different data types (the true gradients are in $\mathrm{nT} / \mathrm{km}$ or nT/degree). Considering the nature of the residuals, and their variations in space and in time, taking the difference between the data from the lower pair of satellites in the East-West direction, spaced about 1.4 degree apart (at the geographic equator, see Friis-Christensen et al., 2006), appears to be an efficient way to mitigate the contributions of large-scale non-lithospheric fields. This gradient however is incomplete since the across difference between the two satellite measurements approximates only the three gradient components along the East-West direction. There is no redundancy with the vector measurements that are still needed to recover the lithospheric field and to stabilize the inverse problem.

The gradient data are not corrected further and we remove the duplicate gradient data that were introduced by considering an overlap of $10^{\circ}$ between the mid and the polar areas. In contrast, three additional dedicated corrections are applied to the vector data. Dedicated corrections applied only to portions of the sphere are necessary to filter out the large-scale residuals shown in Fig. 3. However, such alongtrack corrections require some care as they suffer from spectral leakage due to the impossible separation of the various source fields (see Sabaka and Olsen, 2006; Olsen et al., 2010c). This difficulty is illustrated, for instance, by the expression of the zonal external field coefficient $\widetilde{q}_{l}^{0}$ that would be estimated from the magnetic field vector data measured only along a complete meridian and that would then be used to correct the data along this meridian. It can be shown that (Thébault et al., 2012)

$$
\widetilde{q}_{l}^{0}(\varphi, r)=q_{l}^{0}+\frac{1}{2 l+1} \sum_{n=1}^{\infty} \sum_{m=0}^{n} b_{n}^{m, l}(\varphi, r),
$$

where $q_{l}^{0}$ is the true external coefficient, and $b_{n}^{m, l}$ is a combination of all internal and external residual fields (expressed in $\mathrm{SH}$ ) that contribute to the magnetic field values along the meridian at longitude $\varphi$ and radius $r$. The use of the coefficient $\widetilde{q}_{l}^{0}$ estimated along a single orbit for correcting the magnetic field measurements for the zonal external field is inaccurate and risky depending on the magnitude of $b_{n}^{m, l}(\varphi, r)$. A reduction of this bias is achievable by performing a maximum of prior analysis over the entire globe in order to minimize all internal and external field contributions along all satellite orbits. The along-track correction on the residual data is then applied as a final step.

The first additional block process aims at correcting globally for the large spatial scale error of reduction, be it of internal or external origin. The internal and external Gauss coefficients are estimated to $\mathrm{SH}$ degree 8 in the inclined dipole reference frame with a one year knot-spacing over the full simulated mission duration. We also scale the measurements by the F10.7 solar radio flux values in order to account for the dependence of the ionospheric field on the solar activity. The improvement brought by this scaling is rather marginal but reduces the offsets between close satellite encounters. Note that the internal and external Gauss coefficients estimated by the dedicated corrections have little scientific value as they contain numerous sources of magnetic fields. They are not intended to be interpreted but they remain useful for better understanding the origin of the 
large scale field residuals. There is no major technical limit for this processing block to accept more sophisticated parameterization or improved time resolution. For instance, it could be fine-tuned to absorb most of the remaining large scale magnetic fields in case we lack the explicit models for the main and the magnetospheric fields described above.

A second dedicated correction in spherical harmonics is next applied along the satellite orbits separately for the mid and the polar latitude data. This along-track correction is an important step (e.g., Maus et al., 2006) because the external field perturbations that contain relatively long wavelengths along a satellite track (during magnetically quiet times) change from one orbit to the next. It is worth stressing that some errors are due to uncertainties and changes in the Dst baseline (see Olsen (2002) and Thomson and Lesur (2007) for instance). These introduce lateral spatial oscillations that overlap with the high degree lithospheric field and distort mostly the sectoral harmonics (degrees $n=m$ ). Fortunately, one can further lessen such bias along satellite tracks (Eq. (6)) by applying the correction on large portions of orbits (Thébault et al., 2012). Therefore, all satellite tracks containing segments longer than about $2000 \mathrm{~km}$ without data are rejected. Then, an auxiliary lithospheric field model (AUX_LIT_2 in Fig. 2; taken here from the series of MF models, e.g., Maus et al., 2008) is subtracted from the remaining data. These precautions mitigate the problem of spectral leakage and avoid filtering out too much genuine lithospheric field signals (although this is generally not guaranteed; see figure 5 in Maus et al., 2006). In a synthetic simulation, Sabaka and Olsen (2006; their figures 10 and 11) illustrated the difficulty of recovering all magnetospheric field coefficients up to degree 3 and order 1 at a high time frequency. We exploit this a priori information for tailoring the along-track correction module and we estimate the coefficients of an external field to degree 3 and order 1 plus its induced internal part along each track using simultaneously Swarm A and B measurements. Note that the co-estimation for Swarm A and B represents a significant improvement over estimating the correction along Swarm A and B separately (the bias $b_{n}^{m, l}(\varphi, r)$ being longitude dependant in Eq. (6), it does not leak in a similar way along the Swarm A and B orbits). This estimated external field is then applied as a second correction.

The final dedicated correction is performed via a Fast Fourier Transform along the satellite tracks. The technique of high-pass filtering along the tracks dates back to the 1980's (Langel and Hinze, 1998, section 4.4). The inconvenience of this procedure is that there is no clear cutoff frequency in the signal to isolate the contributions of the lithospheric field without ambiguities (see for instance Wang, 1987 for a discussion). Also, it can only be applied independently on the three magnetic field components. This has much less physical justification than methods based on $\mathrm{SH}$ representation. However, the along-track Fourier filtering has interesting convergence properties. When the data are properly tapered, it significantly filters the remaining intermediate wavelengths while producing little edge effect. We use this processing block to further reduce the magnetic field contributions with length-scales larger than $10^{\circ}$ in arclength along the satellite orbit using a Butterworth filter with a sharp cutoff (filter of order 20). The overlapping data at the junction between mid and polar areas are then used to confine possible edge effects outside the effective data geographic intervals. It is worth stressing that this processing block must be used with extreme care as it cannot discriminate between perturbation and lithospheric fields. In particular, the cut-off frequency must be adjusted depending on how well the AUX_LIT_2 model values temporarily removed from the data agree with the measurements in the filtered waveband.

Finally, the redundant data that were kept to improve the continuity between the processing in mid and polar latitudes (see Subsection 3.1) are removed from the dataset. Then, the standard deviation and the mean of the residuals are computed for each vector component. All values outside the $3 \sigma$ range are considered as outliers and thus rejected and the auxiliary lithospheric field model values subtracted before the series of dedicated corrections are added back.

Figure 4 shows the residual map for the vertical component after all corrections (where, again, the lithospheric field contributions have been subtracted for the purpose of illustration). The processing applied to the vector data is heavy but successful (compare Figs. 3 and 4). However, the corrected measurements now contain a certain amount of artifacts caused by the ad hoc corrections we applied. This shows how important the gradient data are to balance the correction errors made when correcting the vector data for other contributions than those from the lithospheric field. Still, the corrected data can be assumed to reflect mainly the lithospheric field contributions and are now ready to be used for modelling purposes.

\subsection{Regional iterative modelling of the pre-processed data}

The selected and corrected vector data, with their EastWest gradients, are processed regionally (Processes 5 and 6 in Fig. 1). The first step consists in distributing the data within each of the 600 spherical cones that patch the Earth at the Swarm A and B altitudes. Then, the inverse problem is solved for each region. The cones have $8^{\circ}$ aperture, are centered on the nodes of an equal area grid (Saff and Kuijlaars, 1997) about $8.5^{\circ}$ spaced apart, and therefore overlap with each other. The lower and upper radii are automatically defined by the minimum and maximum altitudes of the available data within each cap. The estimated maximum likelihood regional parameters of each type $\bar{G}^{m}$. (see Eqs. (1) and (2)) are stored for each region. The maximum horizontal spatial resolution is about $130 \mathrm{~km}$ (see Eq. (5) with $k_{\max }=15$ ).

The R-SCHA technique, initially set up for processing the CHAMP satellite data (Thébault, 2006), was further developed to ingest the Swarm satellite vector and scalar data. This required the implementation of specific tools to deal also with the magnetic field satellite track differences (referred to as gradient data), with the inversion procedure, its weighting, and its regularization.

Indeed, the gradient and vector data have (here) the same units but they do not have the same statistics. We implemented the possibility of incorporating data error covariance in the inversion. Formally, the noise distribution in the gradient data results from the addition (or subtraction) of 

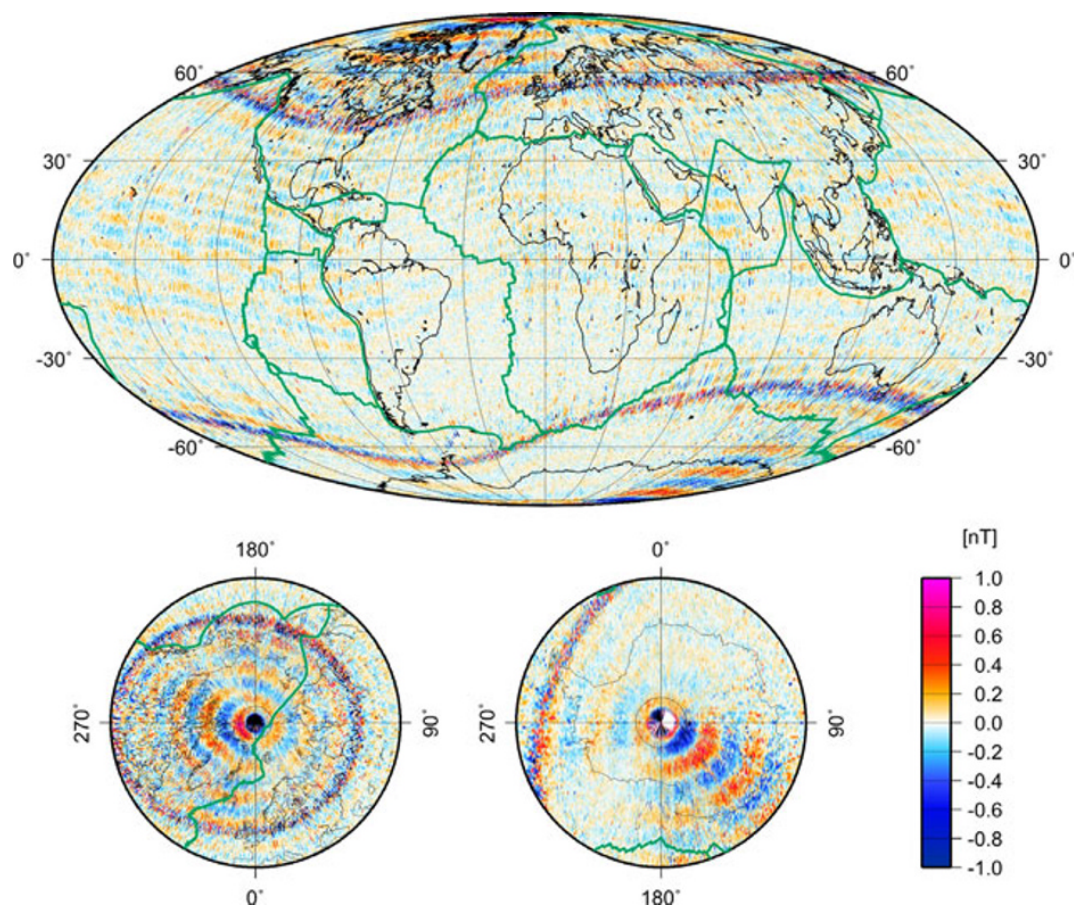

Fig. 4. Vertical component of the residuals after the completion of process 4. The benchmark lithospheric field values have been subtracted for the purpose of this illustration. Mollweide projection.
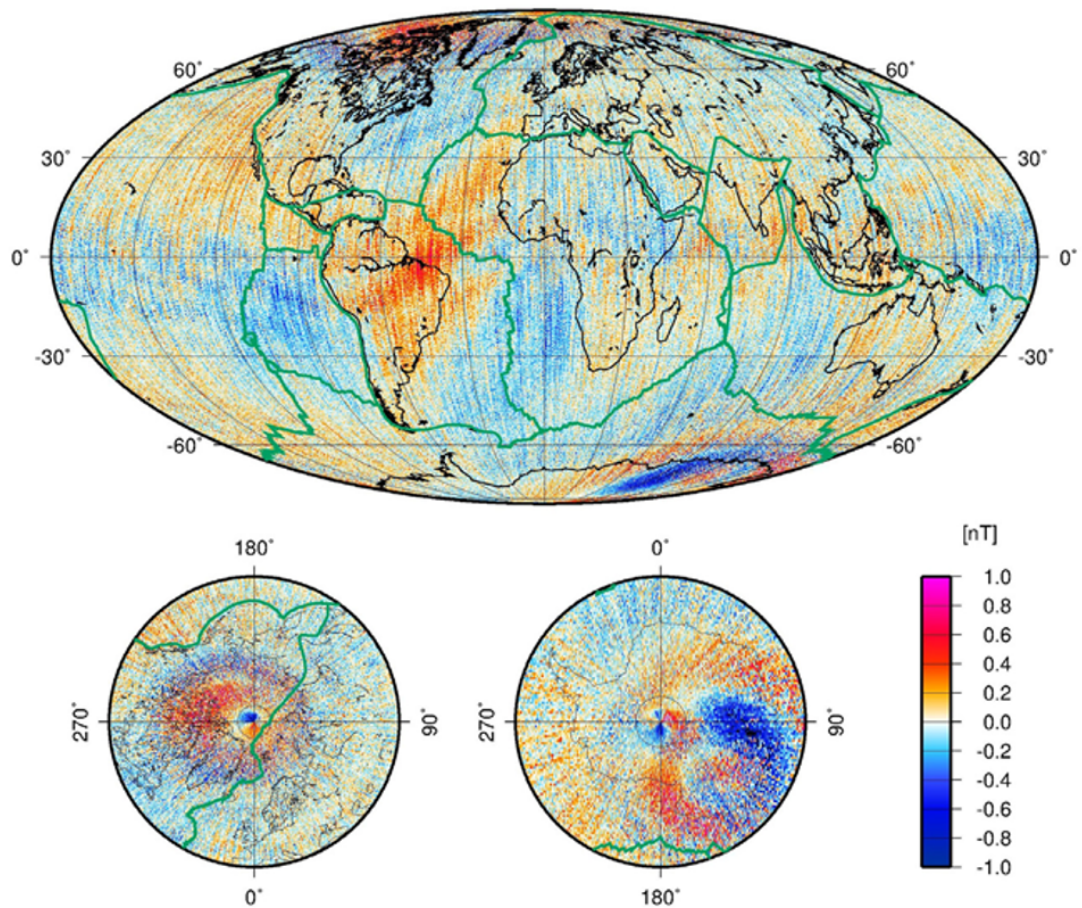

Fig. 5. Vertical component of the difference between the gradient (estimated by finite difference between Swarm A and Swarm B data, see the text for details) that would be estimated using lithosphere only data and the gradient obtained from the data imperfectly corrected for the main internal and external source fields. Projection is Mollweide.

noise in the Swarm A and B measurements. If this noise is Gaussian with a standard deviation of $\sigma_{v}$, the gradient noise would also be Gaussian with a standard deviation of $\sigma_{g}=\sqrt{2} \sigma_{v}$. For the synthetic simulation, the level of instrumental noise added to the synthetic measurements was low enough to neglect this statistical difference. However, it illustrates why we should not expect noise in the gradient and vector data to behave similarly. In addition, the longitudinal distance of $1.4^{\circ}$ between the parallel orbits of Swarm $\mathrm{A}$ and $\mathrm{B}$ was devised to maximize the gain of the gradient data for SH degrees 120-130 (figure 4 in Friis-Christensen et al., 2006). The counterpart is that they have a low gain for low SH degrees. But since the lithospheric field signal measured from space is dominated by the low SH degree contri- 

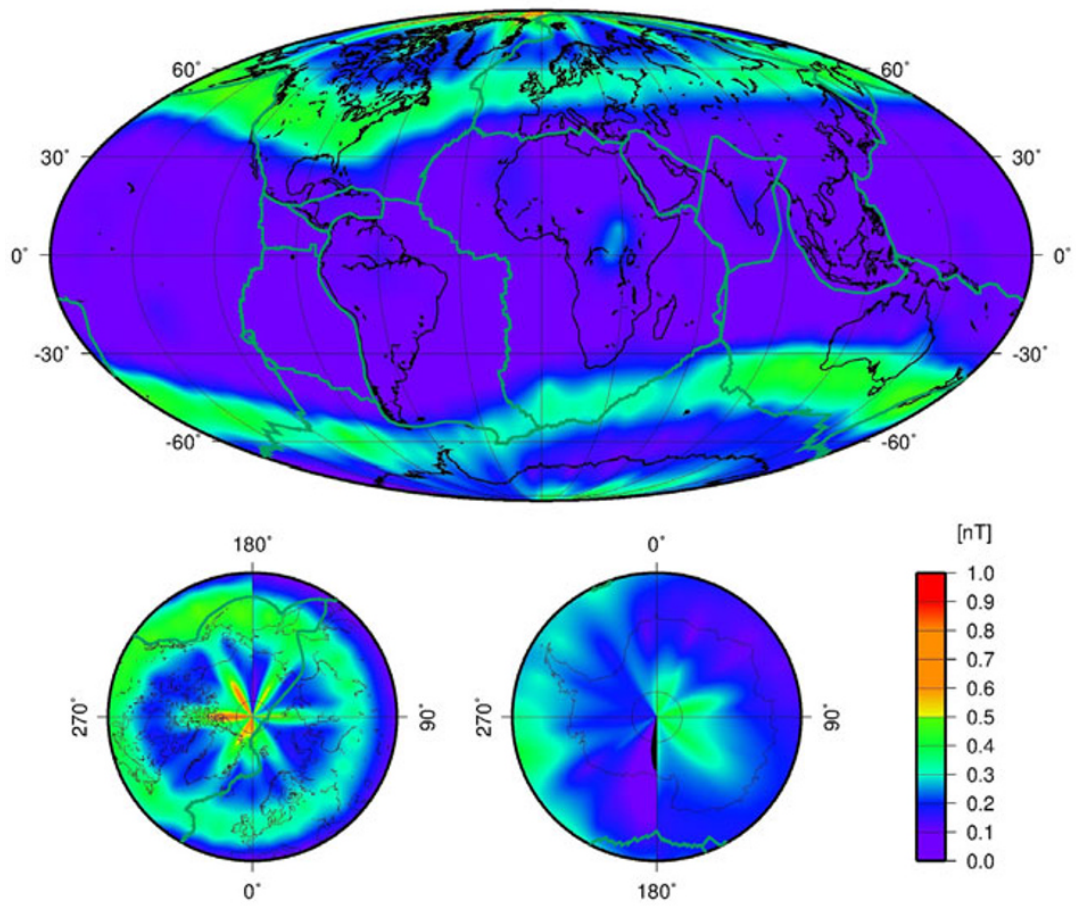

$[\mathrm{nT}]$

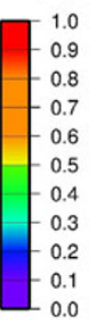

$180^{\circ}$

Fig. 6. Geographical distribution of the misfit after completion of the block processing dedicated to the regional inversions. Mollweide projection.

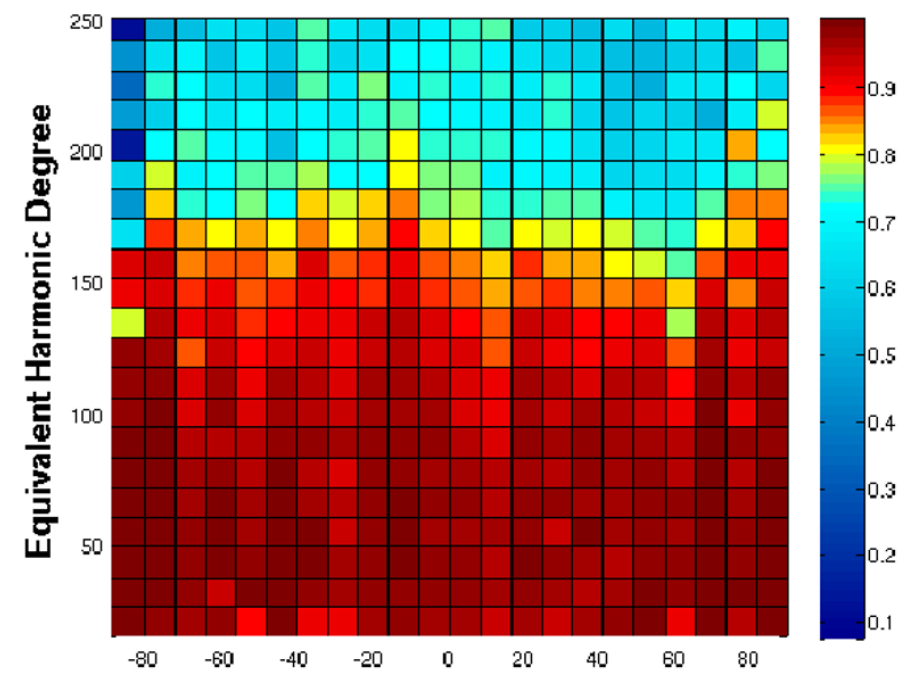

Fig. 7. Spherical Cap Correlation Analysis. The correlation coefficient $\rho_{n_{k}}$ (Eq. (9)) between the regional parameters estimated with lithosphere magnetic field data only and the parameters estimated with all source measurements for each spherical cap degree $n_{k}$ is shown for each band of geographic latitude.

butions (large spatial scales), the root mean square (RMS) of the gradient data is reduced compared to the RMS of the vector data. In terms of the inverse problem, this is an important statement since the functional to be minimized in a least-squares sense is

$$
\chi^{2}=\sum_{n=1}^{N v} \delta B^{2}+\alpha \sum_{n=1}^{N v / 2}\left(\delta \nabla_{\varphi} B\right)^{2}
$$

where $\delta B$ and $\delta \nabla_{\varphi} B$ are the differences between the regional model and the vector and gradient data, respectively (in the east-west direction), and $\alpha$ is a real positive parameter. The parameter $\alpha$ can be tuned to increase the rela- tive weight of gradient data in order to balance the fact that their RMS is lower than the RMS of the vector data and that their number is on average half the number of vector data. However, one must also keep in mind that in principle the vector measurements carry all the lithospheric field information whereas the gradient, being incomplete (we only estimate the East-West gradient), do not. In a thorough prospective work investigating the benefits of gradiometry from space, Kotsiaros and Olsen (2012) indeed illustrate that the East-West gradient data poorly constrain the low SH orders $m$ at all SH degree $n$ (figure 2 in Kotsiaros and Olsen, 2012) showing that vector data are essential to constrain the model. Too much down-weighting of the vector 
data can lead to an ill-posed inverse problem. From a practical viewpoint, it can be seen in Fig. 5 that the corrections for the explicit main and external field models being imperfect, the gradient of the lithospheric field is also affected by correction errors (note the similarity between the structures seen in Figs. 3 and 5). These correction errors are different from those seen in the vector data (Fig. 4). It thus appears that both corrected datasets have different and maybe contradictory information contents. The issue, difficult to address, is to assess which of the contamination affecting the gradient data, and of the reduction errors affecting the vector data, is the largest.

These formal and experimental differences will need to be considered carefully on a case by case basis when the Swarm mission will be in operation. For these reasons, the block processes 6.1 and 6.2 (Fig. 1) solve the least-squares inverse problem independently for each spherical cap

$$
\mathbf{G}=\left(\mathbf{A}^{T} C_{d}^{-1} \mathbf{A}+\lambda \mathbf{R}\right) \mathbf{A}^{T} \mathbf{d}
$$

where $\mathbf{G}$ is the column matrix of the regional parameters to be recovered (Eqs. (1) and (2)) A the matrix of basis functions for the vector and gradient data, $C_{d}^{-1}$ the covariance matrix of the vector and gradient data, $\mathbf{d}$ the vector of magnetic field measurements and their gradients, and $\mathbf{R}$ a regularization matrix multiplied by a scalar Lagrange parameter $\lambda$. Finding the best trade-off between mitigating the data noise, rejecting the correction errors, and exploiting the information content of each dataset is not trivial. We did not try to tackle this problem in the present simulation. We set the covariance matrix to identity and neglect the fact the gradient and the vector data errors could be correlated. The regularization matrix $\mathbf{R}$ can take various forms, depending on the a priori information one would like to exploit regionally, but was left empty. A solution could also be reached in a L1-norm sense by applying an iteratively re-weighted least-squares algorithm with Huber weights in order to obtain a robust solution by iteratively weighting the effect of outlier data in the parameter estimation. We performed several tests and came to the conclusion that this option should be handled with care at the regional scale when processing lithospheric magnetic field measurements. Applying one iteration appears to be sufficient in many cases to reach an optimum solution while not smoothing genuine lithospheric field signal too much. Figure 6 shows the geographical distribution of the residual mean square after completion of the dedicated regional inversion block (Fig. 1). As can be seen most errors are to be found near the polar gaps and around the transitions between mid and polar latitudes that introduce discontinuities in the magnetic field values.

\subsection{Conversion to a SH model and assessment}

For comparison with other lithospheric field models and a more stable continuation of the predictions to the Earth's surface, it is convenient to represent the final lithospheric field model in the form of a set of SH Gauss coefficients. Before we convert the local models into a set of global Gauss coefficients, it is interesting to analyze statistically how close the estimated regional models are to their expected values. By analogy with the spherical harmonics, the degree correlation between two spherical cap harmonic analyses can be estimated by (e.g., Langel and Hinze, 1998; section 4.5.2)

$$
=\frac{\sum_{n_{k}}}{\left\{\left[\sum_{m=0}^{K_{\max }}\left(G_{k}^{m}\right)^{2}+\left(H_{k}^{m}\right)^{2}\right]\left[\sum_{m=0}^{K_{\max }}\left(\widetilde{G}_{k}^{m}\right)^{2}+\left(\widetilde{H}_{k}^{m}\right)^{2}\right]\right\}^{1 / 2}}
$$

with $\left(\widetilde{G}_{k}^{m}, \widetilde{H}_{k}^{m}\right)$ and $\left(G_{k}^{m}, H_{k}^{m}\right)$ the estimated and 'true' local parameters of the Legendre basis function. A similar correlation analysis could be performed with the two other sets of coefficients (The Mehler and the SH ones, see Eqs. (1) and (2)) and, strictly speaking, the quality information provided by this indicator is incomplete. However, only the coefficients of the Legendre basis functions can be related to an equivalent SH degree $n_{k}$ (Eq. (4)) and to a minimum horizontal spatial wavelength (Eq. (5)). The 'true' local parameters are obtained by inverting perfect lithospheric magnetic field measurements containing SH contributions to degree 180. Figure 7 shows the degree per degree correlation analysis sorted in geographic latitude. The correlation is often better than 0.8 up to equivalent $\mathrm{SH}$ degree ranging between 130 and 140 but it degrades more rapidly in the region where mid and high-latitude data were separated (between $\left|40^{\circ}\right|$ and $\left|60^{\circ}\right|$ latitude). Note also that the correlations are in general lower for the R-SCHA degrees between 10 and 30 as a result of the large-scale residual external fields overlapping with the lithospheric field (these large scale fields are visible in the gradient data, see Fig. 5). The message conveyed here is that the regional parameters could be in some places, mostly at mid-latitudes, reliably estimated to a high equivalent spatial degree $n_{k}$.

We finally convert the regional models into a set of Gauss $\mathrm{SH}$ coefficients. The most efficient way to do this is to exploit the sampling theorem on the Earth's sphere (see for instance Schaeffer, 2013 and references therein). We first compute the forward problem for each regional model on the nodes of a Gauss-Legendre grid in the geocentric reference frame (see process 7 in Fig. 1) at $400 \mathrm{~km}$ altitude (this corresponds to the median altitude of the considered dataset). This grid satisfies the sampling theorem to $\mathrm{SH}$ degree 300. To ensure that the numerical solution is not affected too much by the lack of measurements in the polar gap, we rely on a final module. Indeed, large errors, or lack of data, near the geographic pole have a clear adverse effect on the recovery of some constituents of the lithospheric field. The zonal SH coefficients (of order $m=0$ ) are mostly constrained by the magnetic field vector components (mostly the horizontal ones) at high latitudes. The Swarm orbital configuration tested in this study has a polar gap of about $5.2^{\circ}$. This represents a diameter of about 615 $\mathrm{km}$ at $400 \mathrm{~km}$ altitude. Discontinuities caused by the polar gaps introduce ringing when local functions are used. This problem is addressed using a post-processing based on an iterative regional modelling of the vector values computed on the Gauss-Legendre grid above the two polar gaps. The vector data outside the Northern (respectively the Southern) polar gap are first used to derive a low spatial resolution 


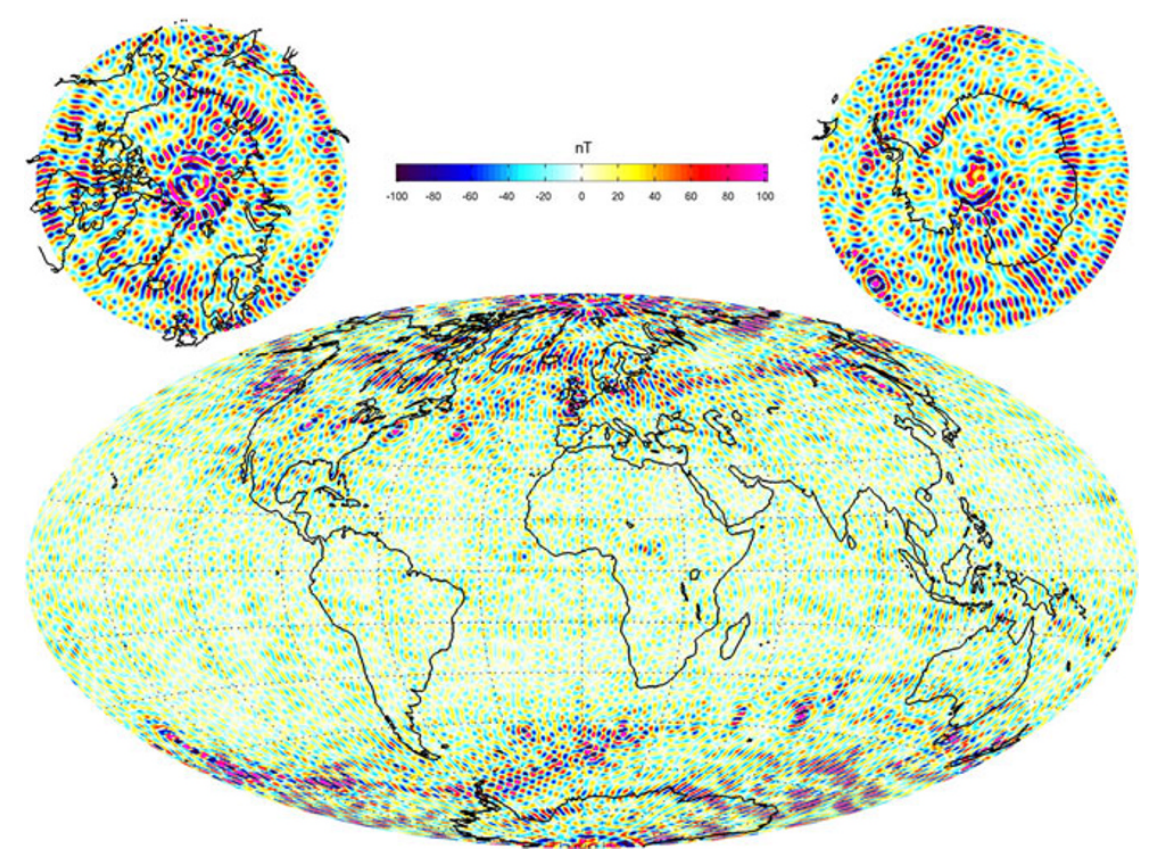

Fig. 8. Residual map of the vertical component between the 'true' and the estimated lithospheric field model at the Earth's mean radius. The models are calculated to SH degree 150. Projection is Mollweide.

regional model (i.e., with a minimum spatial wavelength larger than the size of the polar gap). Synthetic data are then generated from this low resolution model on the GaussLegendre points located within the Northern (respectively the Southern) polar gap areas and a new set of model parameters is estimated at the next iteration to a higher spatial resolution. The process stops when the spatial resolution of the model over the polar gaps reaches the same resolution as in other regions. This iterative procedure could have been implemented directly in the block dedicated to the regional inversions (Fig. 1). However, implementing it as a post-processing step offers a better control because the ratio between the number of gridded data inside and outside the polar gaps is then the same from one simulation to the other.

Finally, we cast the spatial vector data computed on the Gauss-Legendre grid into the SH using a fast spherical transform of the vertical component (process 8 in Fig. 1). This is generally done up to $\mathrm{SH}$ degree 300 to provide a theoretical spatial resolution for the model in agreement with the one provided by the local basis functions. The transformation into SH Gauss coefficients could also be done by a slower module that computes the problem in a leastsquares sense. This process would allow considering the three vector field components, some global scale regularization to be introduced if needed, and to double check the model obtained by the fast spherical transform. The official and final results will then be provided as a set of Gauss coefficients up to SH degree 150 (MLI_SHA_2D, see Fig. 1). However, recognizing that some regions are better represented than others (see Fig. 6), a similar set up to SH 200 (MLI_SHA_2E) will also be provided. This latter model, however, should not be downward continued to the Earth's surface unless otherwise stated (for this reason, we call it a map in Fig. 1).
The performance of the lithospheric field model expressed in SH can be evaluated through four different criteria defined during the preparation of the Swarm mission (Swarm Level 2 Processing System Consortium, 2013). The first one shown in Fig. 8, relies on a geographic map showing the residuals between the true and the estimated lithospheric field models to SH degree 150 at the Earth's mean radius. As can be seen most of the errors are found near the polar gaps and at the mid to high-latitude transitions. More quantitatively, the performance can be estimated using the three complementary criteria in the spectral domain (Fig. 9-left): the spatial power spectrum (Lowes, 1966) of the difference between the 'true' and the estimated lithospheric field model, the spherical harmonic correlation analysis between both models as defined by Eq. (9) for integer degrees $n$ (Fig. 9-middle), and the azimuthal power spectra that provide information about the energy distribution along the orders $m$ (Fig. 9-right). The power spectra and the correlation analysis are presented with the lithospheric field model estimated prior and after the implementation of the post-processing in the polar gaps. As can be seen, this simple expedient significantly improves the model around the order $m=0$. Finally, the resolution matrix between the 'true' and the model estimated with the post-processing for the spectral gaps can also be computed. It tells us that the error is not distributed along a preferred direction except along order $m=0$ (Fig. 10). This is no surprise since neither the vector nor the gradient data are able to constrain these spherical harmonic contributions (see the discussion above). The synthesis of these quality indicators suggests that the model was reliably recovered at least up to SH degree 133, which was the target of this Swarm end-to-end simulation. 

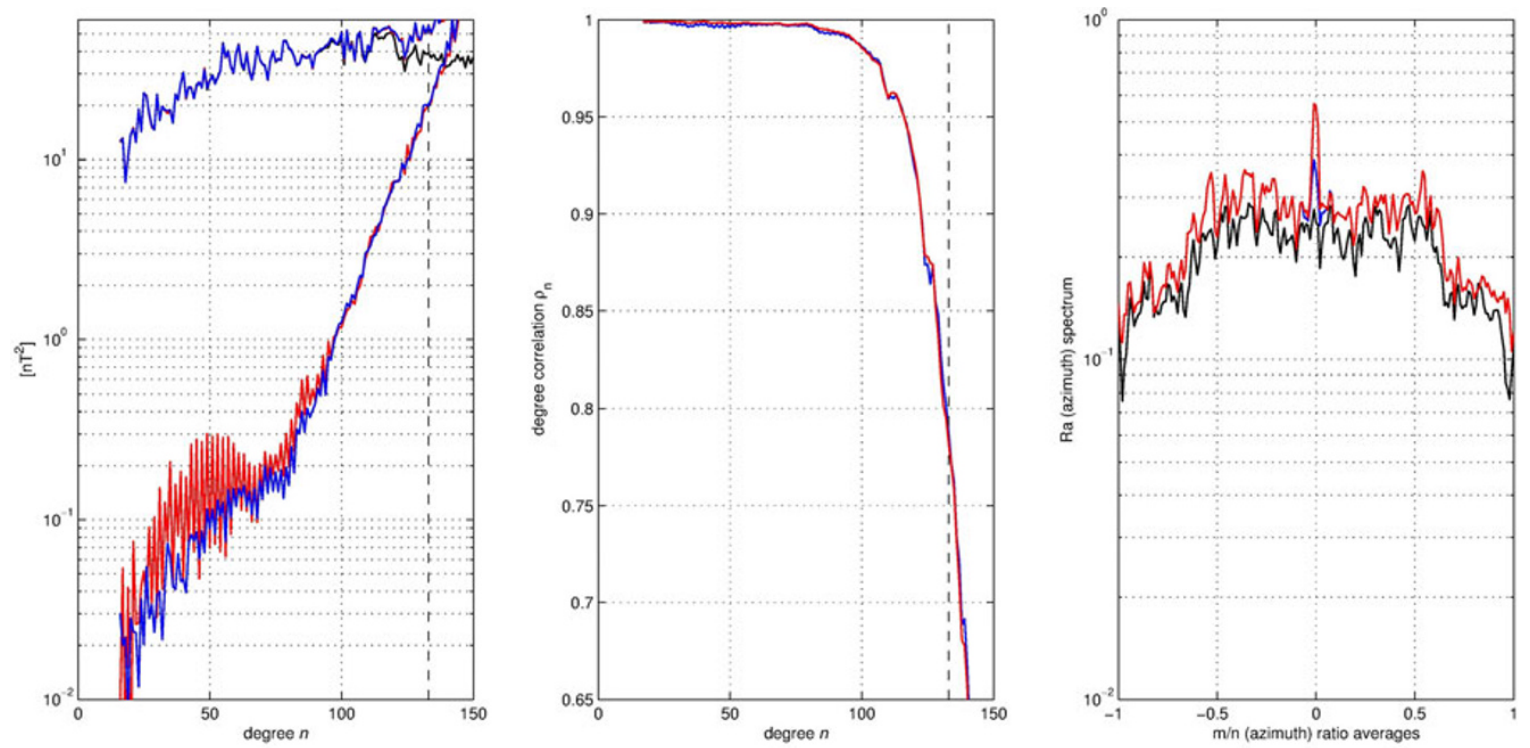

Fig. 9. Power spectral analysis and degree coherence between the 'true' and the estimated lithospheric field models. Left: power spectrum of the 'true' model (black), the estimated model without polar gap treatment (red), and the estimated model with the polar gap processing (blue). The spatial power spectrum of the difference between both types of models and the 'true' one are also shown. Middle: Spherical Harmonic Correlation Analysis between the 'true' model and the model estimated with (blue) and without (red) polar gap post-processing. The dashed vertical line indicate SH degree 133. Right: Azimuthal power spectrum of the 'true' model (black) and the model estimated with (blue) and without (red) polar post-processing.

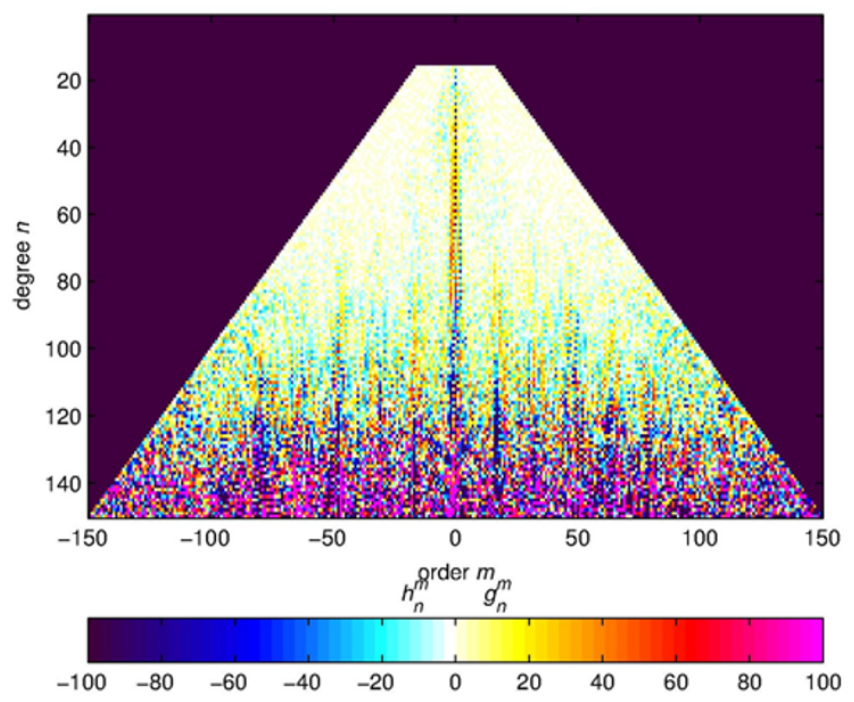

Fig. 10. Sensitivity matrix between the 'true' model and the model estimated after a polar gap processing.

\section{Conclusions}

In this paper we presented the Swarm SCARF Dedicated Lithospheric Field Inversion chain, which will be used to process the magnetic data of the forthcoming Swarm satellite mission and to produce a model for the Earth's lithospheric field. This approach differs significantly from the comprehensive approach (Sabaka et al., 2013) as it heavily relies on data selection, corrections, and visual inspections. It also uses a modeling scheme based on local functions that were designed to better detect the small scales of the lithospheric field. During the operational phase, the criteria discussed in this paper will be subject to changes and will therefore be described in a companion report of the Swarm SCARF dedicated lithospheric field model. With the current setting, the algorithm provides a model that meets the required performance at least up to $\mathrm{SH}$ degree 133 when considering and the synthetic data provided to us for the full mission duration. This is a better spatial resolution than achieved so far by single satellite missions at comparable altitudes. This result confirms that we are able to develop strategies for recovering the lithospheric magnetic field to a high spatial resolution in a favorable situation when high quality vector magnetic field measurements are available for the two lower Swarm A and B satellites. This simulation was realistic in the sense that it was realized with a mathematical method different from the one used to synthesize the simulated data, with a separation into regions that inevitably introduces discontinuities, with imperfect main and external field models for correcting the synthetic measurements for non-lithospheric magnetic fields, and with real 
proxies for selecting the data for quiet magnetic days along the entire simulated mission duration. However, in the operational phase, the performance of this chain will depend on our ability to estimate the real quality of the input magnetic field measurements and the accuracy of the scientific products used to correct for the main and the external fields. For instance, complementary simulations (not shown) performed with the same parameters as the ones described in this paper suggest that a small bias of $0.3 \mathrm{nT}$ affecting intermittently Swarm A or B vector measurements, would degrade the overall performance down to about $\mathrm{SH}$ degree $110-120$. It is worth stressing that even this degraded performance remains impressive compared to those achieved by single satellite missions, which based on the experience with CHAMP are of the order of $\mathrm{SH}$ degree 85 (this it the maximum SH degree for which models start being in significant disagreement). An improvement from $n=85$ to $n=120$ means that twice as many SH Gauss coefficients would be recovered (the number of Gauss coefficients is $n(n+2))$. This spatial resolution limit may not be a hard bound but it shows that the recipe we followed may need to be revised and fine-tuned when real data will become available.

The Swarm calibration/validation phase will be a valuable learning experience to better control the intrinsically different information content and contamination errors of the vector and the gradient data. Some tools have been developed to account for the statistical differences between these two types of datasets. In this paper, we did not try to optimize the weights or to regularize the inverse problem but this possibility was left open on both the regional (Process 6.2) and the global scales (Process 8). More theoretical work will be needed to define the most relevant physical norms to be used for the lithospheric field and fully benefit from this possibility. We may also select the spectral band corresponding to the highest information content for each type of data to constrain the model with vector data at the low SH degrees and with gradient data at high $\mathrm{SH}$ degrees (see Kotsiaros and Olsen, 2012 for a discussion). This is an option we did not consider at present because the gradient estimated from the Swarm lower pair of satellites is not complete. Its implementation is not necessarily complex but it adds another level of subjectivity concerning the respective spectral bands that are better constrained by each data type.

A major issue we did not address is that of the impact on the East-West gradient of undesired magnetic signals due to electric currents that would cross the two orbits of the lower pair of satellites. At present, the R-SCHA technique is efficient for processing poloidal magnetic field measurements but cannot handle toroidal fields without some significant amount of development. This is the main shortcoming of the Dedicated Lithospheric Field Inversion chain in its present form. But we may still exploit the information provided by the East-West gradient to at least estimate the amount of current crossing the Swarm A and B mesocenter (see Kotsiaros and Olsen, 2012, equation (3.8); or Shen et $a l ., 2012$, for instance) and to use this as a selection criterion. We could then use the gradient at times (and location) of negligible estimated currents. We note that, as it stands, the chain could also readily be used with scalar gradient data only that are in general less affected by toroidal fields.

One key advantage of the Dedicated Lithospheric Field Inversion chain is that it is based on a regional modeling approach. This allows the inverse problem to be solved independently for each region. Depending on the available computer resources, the process divided in as many independent jobs is intrinsically fast. For the present simulation, a few hours were necessary to select and process more than five million out of more than $250.10^{6}$ available vector synthetic measurements of the Swarm A and B satellites. A fast algorithm is advantageous considering the chosen philosophy. Isolating the lithospheric field relies on a careful selection of data and on compromises made in correcting the data more or less empirically. Therefore, fast processes open an avenue for computing parades of statistically equivalent models in order to estimate the variability of each $\mathrm{SH}$ degree part depending on the applied selections and corrections. An immediate application of this principle would be to play with the boundary separating mid and polar regions as it introduces one of the largest source of errors in this simulation (see Fig. 8).

The algorithm is also highly modular. Complementary processing blocks could be included as more and more experience is gained with the real Swarm satellite measurements. Preprocessing blocks based on quality indicators, flags, and star camera switches could be useful to account for instrumental failures or malfunctions. The algorithm is therefore not frozen and will benefit from the scientific advances prompted by the Swarm mission along its entire duration. Finally, we note that the median altitude of Swarm $\mathrm{A}$ and B measurements was about $400 \mathrm{~km}$ for the simulations we discussed. An extension of the mission to lower altitudes would amplify the small-scale signals, which are currently strongly attenuated at $400 \mathrm{~km}$ altitude, and would possibly allow us to derive lithospheric field models to even higher resolution.

Acknowledgments. The authors gratefully acknowledge support from the Centre National des Etudes Spatiales (CNES) within the context of the "Travaux préparatoires et exploitation de la mission Swarm" project and from the European Space Agency (ESA) through the ESTEC contract No. 4000102140/10/NL/JA "Development of the Swarm Level2 Algorithms and Associated Level2 Processing Facility". We also acknowledge the comments of two anonymous referees that helped to improve the manuscript. This is IPGP contribution number 3410 .

\section{References}

Alken, P., S. Maus, P. Vigneron, O. Sirol, and G. Hulot, Swarm SCARF equatorial electric field inversion chain, Earth Planets Space, 65, this issue, 1309-1317, 2013.

Backus, G., R. L. Parker, and C. Constable, Foundations of Geomagnetism, Cambridge Univ. Press, Cambridge, U.K., 1996.

Beggan, C. D., J. Saarimäki, K. A. Whaler, and F. J. Simons, Spectral and spatial decomposition of lithospheric magnetic field models using spherical Slepian functions, Geophys. J. Int., doi:10.1093/gji/ggs122, 2013.

Chulliat, A., P. Vigneron, E. Thébault, O. Sirol, and G. Hulot, Swarm SCARF Dedicated Ionospheric Field Inversion chain, Earth Planets Space, 65, this issue, 1271-1283, 2013.

Finlay, C. C., S. Maus, C. Beggan, M. Hamoudi, V. Lesur, F. J. Lowes, N. Olsen, and E. Thébault, Evaluation of candidate geomagnetic field models for IGRF-11, Earth Planets Space, 62(10), 787-804, 2010.

Fox Maule, C., M. Purucker, N. Olsen, and K. Mosegaard, Heat flux 
anomalies in Antarctica revealed from satellite magnetic data, Science, doi:10.1126, 2005.

Friis-Christensen, E., H. Lühr, and G. Hulot, Swarm: A constellation to study the Earth's magnetic field, Earth Planets Space, 58(4), 351-358, 2006.

Haines, G. V., Spherical cap harmonic analysis, J. Geophys. Res., 90, 2583-2591, 1985

Hemant, K. and S. Maus, Geological modeling of the new CHAMP magnetic anomaly maps using a Geographical Information System (GIS) technique, J. Geophys. Res. B, 110, B12103, doi:10.1029/2005JB003837, 2005.

Hulot, G., N. Olsen, and T. J. Sabaka, The present field, in Volume 5 Geomagnetism, Treatise on Geophysics, vol. 5 (Elsevier, Amsterdam, 2007), pp. 33-72, 2007.

Kotsiaros, S. and N. Olsen, The geomagnetic field gradient tensorProperties and parametrization in terms of spherical harmonics, Int. J. Geomath., 3, 297-314, 2012.

Langel, R. A. and W. J. Hinze, The Magnetic Field of the Earth's Lithosphere: The Satellite Perspective, 429 pp., Cambridge Univ. Press, New York, 1998.

Langlais, B., M. E. Purucker, and M. Mandea, Crustal magnetic field of Mars, J. Geophys. Res., 109(E2), doi:10.1029/2003JE002048, issn: 0148-0227, 2004.

Lesur, V., Introducing localized constraints in global geomagnetic field modelling, Earth Planets Space, 58, 477-483, 2006.

Lesur, V. and S. Maus, A global lithospheric magnetic field model with reduced noise level in the Polar Regions, Geophys. Res. Lett., 33, L13304, doi:10.1029/2006GL025826, 2006.

Lesur, V., I. Wardinski, M. Hamoudi, and M. Rother, The second generation of the GFZ Reference Internal Magnetic Model: GRIMM-2, Earth Planets Space, 62(10), 765-773, 2010.

Lesur, V., M. Rother, F. Vervelidou, M. Hamoudi, and E. Thébault, Postprocessing scheme for modeling the lithospheric magnetic field, Solid Earth, 4, 105-118, doi:10.5194/se-4-105-2013, 2013.

Lowes, F. J., Mean square values on the sphere of spherical harmonic vector fields, J. Geophys. Res., 71, 2179, 1966.

Maier, T. and C. Mayer, Multiscale downward continuation of CHAMP FGM-data for crustal field modelling, in First CHAMP Mission Results for Gravity, Magnetic and Atmospheric Studies, edited by C. Reigber, H. Lühr, and P. Schwintzer, pp. 288-295, Springer Verlag, 2003.

Maus, S., An ellipsoidal harmonic representation of Earth's lithospheric magnetic field to degree and order 720, Geochem. Geophys. Geosyst., 11, Q06015, doi:10.1029/2010GC003026, 2010.

Maus, S., H. Lühr, and M. E. Purucker, Simulation of the high-degree lithospheric field recovery for the Swarm constellation of satellites, Earth Planets Space, 58(4), 397-407, 2006.

Maus, S., F. Yin, H. Lühr, C. Manoj, M. Rother, J. Rauberg, I. Michaelis, C. Stolle, and R. D. Müller, Resolution of direction of oceanic magnetic lineations by the sixth-generation lithospheric magnetic field model from CHAMP satellite magnetic measurements, Geochem. Geophys. Geosyst., 9, Q07021, doi:10.1029/2008GC001949, 2008.

Olsen, N., A model of the geomagnetic field and its secular variation for epoch 2000, Geophys. J. Int., 149(2), 454-462, 2002.

Olsen, N., M. Mandea, T. J. Sabaka, and L. Toffner-Clausen, The CHAOS3 Geomagnetic Field Model and Candidates for the 11th Generation of IGRF, Earth Planets Space, 62, 719-727, 2010a.

Olsen, N., G. Hulot, and T. J. Sabaka, Sources of the geomagnetic field and the modern data that enable their investigation, in Handbook of Geomathematics, edited by W. Freeden, M. Z. Nashed, and T. Sonar, chapter 5, pp. 106-124, doi:10.1007/978-3-642-01546-5-5, 2010b.

Olsen, N., K.-H. Glassmeier, and X. Jia, Separation of the Magnetic Field into External and Internal Parts, Space Sci. Rev., 152, 135-157, doi:10.1007/s11214-009-9563-0, 2010c.

Olsen, N., E. Friis-Christensen, R. Floberghagen, P. Alken, C. D Beggan, A. Chulliat, E. Doornbos, J. T. da Encarnação, B. Hamilton, G. Hulot, J. van den IJssel, A. Kuvshinov, V. Lesur, H. Lühr, S. Macmillan, S. Maus,
M. Noja, P. E. H. Olsen, J. Park, G. Plank, C. Püthe, J. Rauberg, P. Ritter, M. Rother, T. J. Sabaka, R. Schachtschneider, O. Sirol, C. Stolle, E. Thébault, A. W. P. Thomson, L. Tøffner-Clausen, J. Velímský, P. Vigneron, and P. N. Visser, The Swarm Satellite Constellation Application and Research Facility (SCARF) and Swarm data products, Earth Planets Space, 65, this issue, 1189-1200, 2013.

Purucker, M., B. Langlais, N. Olsen, G. Hulot, and M. Mandea, The southern edge of cratonic North America: Evidence from new satellite magnetometer observations, Geophys. Res. Lett., 29(15), article number 013645, doi:10.1029/2001GL013645, 2002.

Reigber, C., H. Lühr, and T. Schwintzer, Champ Mission status, Adv. Space Res., 30(2), 129-134, doi:10.1016/S02731177(02)00276-4, 2002.

Rother, M., V. Lesur, and R. Schachtschneider, An algorithm for deriving core magnetic field models from the Swarm data set, Earth Planets Space, 65, this issue, 1223-1231, 2013.

Sabaka, T. J. and N. Olsen, Enhancing comprehensive inversions using the Swarm constellation, Earth Planets Space, 58, 371-395, 2006.

Sabaka, T. J., L. Tøffner-Clausen, and N. Olsen, Use of the Comprehensive Inversion method for Swarm satellite data analysis, Earth Planets Space, 65, this issue, 1201-1222, 2013.

Saff, E. B. and A. B. J. Kuijlaars, Distributing many points on a sphere, The Mathematical Intelligencer, 19(1), 5-11, 1997.

Shen, C., Z. J. Rong, and M. Dunlop, Determining the full magnetic field gradient from two spacecraft measurements under special constraints, $J$. Geophys. Res., 117, A10217, doi:10.1029/2012JA018063, 2012.

Schaeffer, N., Efficient Spherical Harmonic Transforms aimed at pseudospectral numerical simulations, Geochem. Geophys. Geosyst., 14(3), 751-758, 2013.

Schott, J. J and E. Thébault, Modelling the earth's magnetic field from global to regional scales, in Geomagnetic Observations and Models, IAGA Special Sopron Book Series, Volume 5, 229-264, doi:10.1007/978-90-481-9858-0-9, 2011

Stockman, R., C. C. Finlay, and A. Jackson, Imaging Earth's crustal magnetic field with satellite data: A regularized spherical triangle tessellation approach, Geophys. J. Int., 179, 929-944, doi:10.1111/j.1365246X.2009.04345.x, 2009.

Swarm Level 2 Processing System Consortium, Product specification for L2 Products and Auxiliary Products, Doc.no: SW-DS-DTU-GS-0001, 2013.

Thébault, E., Global lithospheric magnetic field modeling by successive regional analysis, Earth Planets Space, 58, 485-495, 2006.

Thébault, E., J. J. Schott, M. Mandea, and J. P. Hoffbeck, A new proposa for Spherical Cap Harmonic Analysis, Geophys. J. Int., 159, 83-105, doi:10.1111/j.1365-246X.2004.02361.x, 2004.

Thébault, E., J. J. Schott, and M. Mandea, Revised spherical cap harmonic analysis (R-SCHA): Validation and properties, J. Geophys. Res., 111 B01102, doi:10.1029/2005JB003836, 2006.

Thébault, E., K. Hemant, G. Hulot, and N. Olsen, On the geographical distribution of induced time-varying crustal magnetic fields, Geophys. Res. Lett., 36, L01307, doi:10.1029/2008GL036416, 2009.

Thébault, E., M. Purucker, K. Whaler, B. Langlais, and T. J. Sabaka, The Magnetic field of the Earth's lithosphere, Space Sci.Rev., 155(1-4), 95127, doi:10.1007/s11214-010-9667-6, 2010.

Thébault, E., F. Vervelidou, V. Lesur, and M. Hamoudi, The along-track satellite analysis in planetary magnetism, Geophys. J. Int., 188(3), 891907, doi:10.1111/j.1365-246X.2011.05281.x, 2012.

Thomson, A. W. P. and V. Lesur, An improved geomagnetic data selection algorithm for global geomagnetic field modelling, Geophys. J. Int., 169(3), 951-963, doi:10.1111/j.1365-246X.2007.03354.x, 2007.

Wang, Z., Understanding models of the geomagnetic field by Fourier analysis, J. Geomag. Geoelectr., 39, 333-347, 1987.

E. Thébault (e-mail: ethebault@ipgp.fr), P. Vigneron, S. Maus, A. Chulliat, O. Sirol, and G. Hulot 